\title{
Perfusion-weighted magnetic resonance imaging of the brain: techniques and application in children
}

\author{
Huisman, Thierry A G M ; Sorensen, A Gregory
}

\begin{abstract}
Perfusion-weighted magnetic resonance imaging (PWI) has been proposed as an attractive non-invasive tool for evaluating cerebral haemodynamics. Quantitative maps of cerebral blood flow $(\mathrm{CBF})$, cerebral blood volume (CBV), mean transit time (MTT), time to peak (TTP) and various other haemodynamic parameters can be obtained. Recent advances in hard- and software made PWI available for clinical routine. Although PWI became common in adult neuroradiology, it remains challenging in pediatric neuroradiology. In this article, the different PWI techniques that render haemodynamic maps of the brain are presented and discussed. The normal developmental changes of the cerebral haemodynamics in children as measured by PWI are presented as well as the application of PWI in cerebral ischaemia, primary and secondary cerebral vasculopathies and in cerebral tumours
\end{abstract}

DOI: https://doi.org/10.1007/s00330-003-1972-y

Posted at the Zurich Open Repository and Archive, University of Zurich

ZORA URL: https://doi.org/10.5167/uzh-156241

Journal Article

Published Version

Originally published at:

Huisman, Thierry A G M; Sorensen, A Gregory (2004). Perfusion-weighted magnetic resonance imaging of the brain: techniques and application in children. European Radiology, 14(1):59-72.

DOI: https://doi.org/10.1007/s00330-003-1972-y 
Thierry A. G. M. Huisman

A. Gregory Sorensen

\section{Perfusion-weighted magnetic resonance imaging of the brain: techniques and application in children}

Received: 10 June 2002

Revised: 20 March 2003

Accepted: 2 May 2003

Published online: 25 June 2003

(C) Springer-Verlag 2003

T. A. G. M. Huisman ( ) A. G. Sorensen Department of Radiology,

Neuroradiology Section

and MGH-NMR Center,

Massachusetts General Hospital

and Harvard Medical School,

Boston, Massachusetts, USA

e-mail: thierry.huisman@kispi.unizh.ch

Tel.: +41-1-2667111

Fax: +41-1-2667158

Present address:

T. A. G. M. Huisman,

Department of Radiology,

University Children's Hospital Zurich,

Steinwiesstrasse 75, 8032 Zurich,

Switzerland

\begin{abstract}
Perfusion-weighted magnetic resonance imaging (PWI) has been proposed as an attractive noninvasive tool for evaluating cerebral haemodynamics. Quantitative maps of cerebral blood flow (CBF), cerebral blood volume (CBV), mean transit time (MTT), time to peak (TTP) and various other haemodynamic parameters can be obtained. Recent advances in hard- and software made PWI available for clinical routine. Although PWI became common in adult neuroradiology, it remains challenging in pediatric neuroradiology. In this article, the different PWI techniques that render haemodynamic maps of the brain are presented and discussed. The normal
\end{abstract}

developmental changes of the cerebral haemodynamics in children as measured by PWI are presented as well as the application of PWI in cerebral ischaemia, primary and secondary cerebral vasculopathies and in cerebral tumours.

Keywords Perfusion-weighted imaging - Dynamic susceptibility contrast imaging $\cdot$ Haemodynamic parameter $\cdot$ Children

\section{Introduction}

The cerebral circulation relies on a dynamic vascular system that maintains a steady state delivery of blood to the brain. Autoregulatory mechanisms are designed to preserve an adequate, stable cerebral perfusion. The close relationship between cerebral perfusion, metabolism and neuronal function was described as early as 1890 by Roy and Sherrington [1].

Cerebral perfusion is typically measured as the quantity of blood $(\mathrm{ml})$ perfusing a volume of brain $(100 \mathrm{~g})$ per unit of time ( $\mathrm{min}$ ), also known as cerebral blood flow (CBF). Several additional, closely linked parameters, including cerebral blood volume (CBV) and blood circulation time, are used to characterise cerebral haemodynamics. The CBV is the amount of blood in a given amount of brain while the mean transit time (MTT) represents the time the blood takes to pass the brain tissue. These haemodynamic parameters are linked by the following relationship:

$\mathrm{CBF} \times \mathrm{MTT}=\mathrm{CBV}$

Cerebral blood flow is influenced by multiple, complex interacting factors including arterial perfusion pressure, intracranial pressure, blood viscosity, arterial $\mathrm{pCO}_{2}$ and $\mathrm{pO}_{2}$. Cerebral autoregulation mechanisms preserve CBF within a narrow range to guarantee brain tissue integrity and function. The $\mathrm{CBF}$ and $\mathrm{CBV}$ are tightly coupled [2].

Many disease processes are known to alter cerebral perfusion. These changes can be in either direction. Cerebral perfusion can be reduced (cerebral ischaemia) or increased (arterio-venous malformations). In addition, in many disease processes $\mathrm{CBF}$ and $\mathrm{CBV}$ can be decoupled. In acute cerebral ischaemia, for example, a cerebral vasodilatation can occur in compensation to a decreased 
perfusion pressure. This vasodilatation increases CBV, whereas CBF remains below normal $[3,4,5,6]$.

Neuro-anatomical imaging techniques, including CT and MRI, represent important diagnostic tools in the evaluation of cerebrovascular disease (CVD). A major limitation is that these techniques identify only the sequelae from CVD without giving detailed information about the underlying cerebral haemodynamics. In addition, conventional $\mathrm{CT}$ and MRI often detect injury too late, i.e. after injury has become irreversible. An alternative technique that gives functional, haemodynamic information is therefore desirable. This technique should identify the aetiology and severity of the vascular pathology, estimate the risk of future tissue injury, direct the correct treatment, evaluate and monitor the results of treatment and finally give insight in the pathophysiology of disorders involving the cerebrovascular system and haemodynamics in general.

Multiple techniques have been proposed to investigate the cerebrovascular system. Some provide nontomographic, regional data, whereas others provide projectional or tomographic images. Imaging includes X-ray angiography, CT angiography, MR angiography and Doppler ultrasonography. These techniques are, however, focused on detecting blood flow in the macroscopic vasculature, whereas most cerebrovascular diseases begin at the microvascular or capillary level. The macroscopic vasculature is seldom involved in the early stages of disease. Detection of pathology at the microvascular level is consequently favourable because treatment could be started at an earlier stage of the disease, possibly even before irreversible clinical symptoms develop.

Techniques that provide haemodynamic information at the capillary level include positron emission tomography (PET), single photon emission computed tomography (SPECT) and dynamic ${ }^{133}$ xenon CT. These methods are, however, expensive, time-consuming and require the administration of ionizing radiation: a distinct disadvantage in children.

Perfusion-weighted MRI is a tomographic imaging technique that combines unique features in assessing brain perfusion. PWI is relatively sensitive to the microvasculature, is minimally invasive, has a higher spatial resolution than PET/SPECT, does not use ionizing radiation and has a better contrast-to-noise per unit of time compared with X-ray CT. In addition, MRI scanners are more widely available than PET/SPECT scanners, PWI can easily be added to conventional MRI (short acquisition time), and finally the faster heart rate and smaller perfusion cross-sectional area of the child's brain increase the quality of PWI.

\section{How is tissue perfusion measured by MRI}

There are currently two fundamentally different approaches to measure brain perfusion by MRI. The first, most widely applied method is referred to as the "dynamic susceptibility contrast technique". This approach uses long-established principles of indicator dilution methods in which a decrease of T2 or T2* MR signal intensity is related to the passage of paramagnetic contrast materials through the capillary bed $[7,8,9]$. The drop in MR signal intensity gives information about multiple haemodynamic parameters.

The second, currently less often applied method relies on the use of inversion or saturation pulses that label blood spins before flowing into the brain slice of interest. In this technique, also known as "arterial spin labelling" (ASL), the consequent MR signal changes are directly related to the local haemodynamics.

A third MRI technique that uses phase-sensitive sequences to capture MR signal changes due to perfusioninduced intravoxel incoherent motion (IVIM) is presently mainly of historical importance and is consequently only mentioned for completeness but is not discussed in this paper [10].

\section{Dynamic susceptibility contrast imaging}

Dynamic susceptibility contrast imaging relies on $\mathrm{T} 2$ or T2* signal changes within the brain tissue due to susceptibility effects of high doses of paramagnetic contrast material flowing through the brain vasculature (Figs. 1, 2). Unlike conventional MRI, the basis of PWI does not rely on relaxivity (dipole-dipole) effects but primarily on magnetic susceptibility effects.

The short range at which dipole-dipole effects of paramagnetic contrast agents (e.g. gadolinium chelates) act, restrict their effects upon those spins which are in direct contact with the contrast agent. Because most routinely used paramagnetic contrast agents do not cross the blood-brain barrier (BBB), these dipole-dipole effects are consequently limited to the intravascular space or cerebral blood pool. This compartmentalization means that if we would rely on these dipole-dipole effects haemodynamic maps would be calculated from maximal $4 \%$ of the cerebral spins.

$\mathrm{T} 2$ and $\mathrm{T} 2 *$ weighted dynamic susceptibility contrast imaging takes advantage of the compartmentalization. The accumulation of high magnetic susceptibility contrast agents within the approximately randomly oriented capillary network of the brain results in localized variations in tissue magnetic fields. This microscopic heterogeneity of magnetic field induces a loss of transverse phase coherence with T2/T2* signal loss in the surrounding brain tissue (outside of the vascular compartment; Figs. 1, 2). Magnetic susceptibility effects act on a longer range than the previously described dipole-dipole in- 
Fig. 1 Gradient-echo, echo-planar (EPI) dynamic susceptibility contrast imaging. A series of raw images after the injection of a bolus of gadolinium is displayed. The first four images show the precontrast baseline signal intensity. The arrival of the gadolinium is first seen within branches of the middle cerebral artery, $6 \mathrm{~s}$ after contrast injection, followed by a marked parenchymal signal loss during the first passage of the gadolinium (9-16.5 s). A phase of rapid signal recovery is then followed due to the first recirculation of the gadolinium $(27-31.5 \mathrm{~s})$. At the end of the series, a discrete residual signal loss compared with the initial baseline images is seen due to remaining circulating contrast agent by a discrete second signal drop

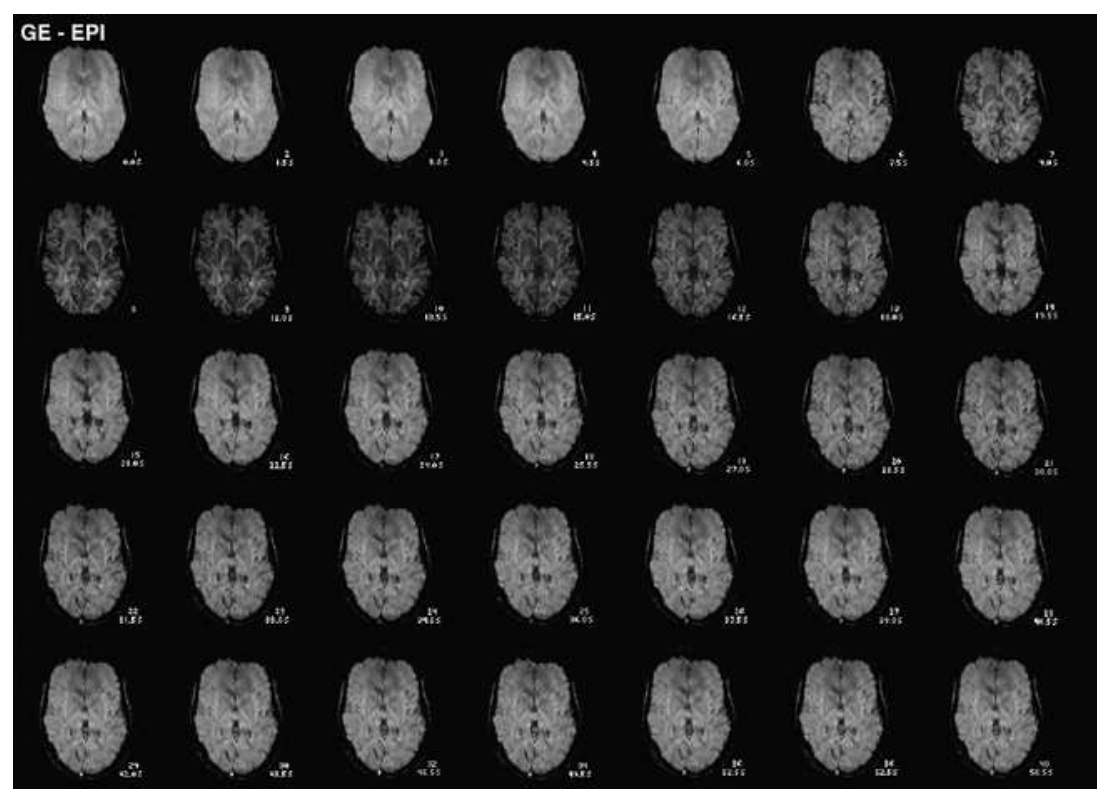

Fig. 2 Spin-echo EPI dynamic susceptibility contrast imaging. A series of raw images during the passage of a bolus of gadolinium is displayed. The signal drop is identical in the temporal evolution; the degree of signal drop is, however, less pronounced compared to Fig. 1 (gradient-echo)

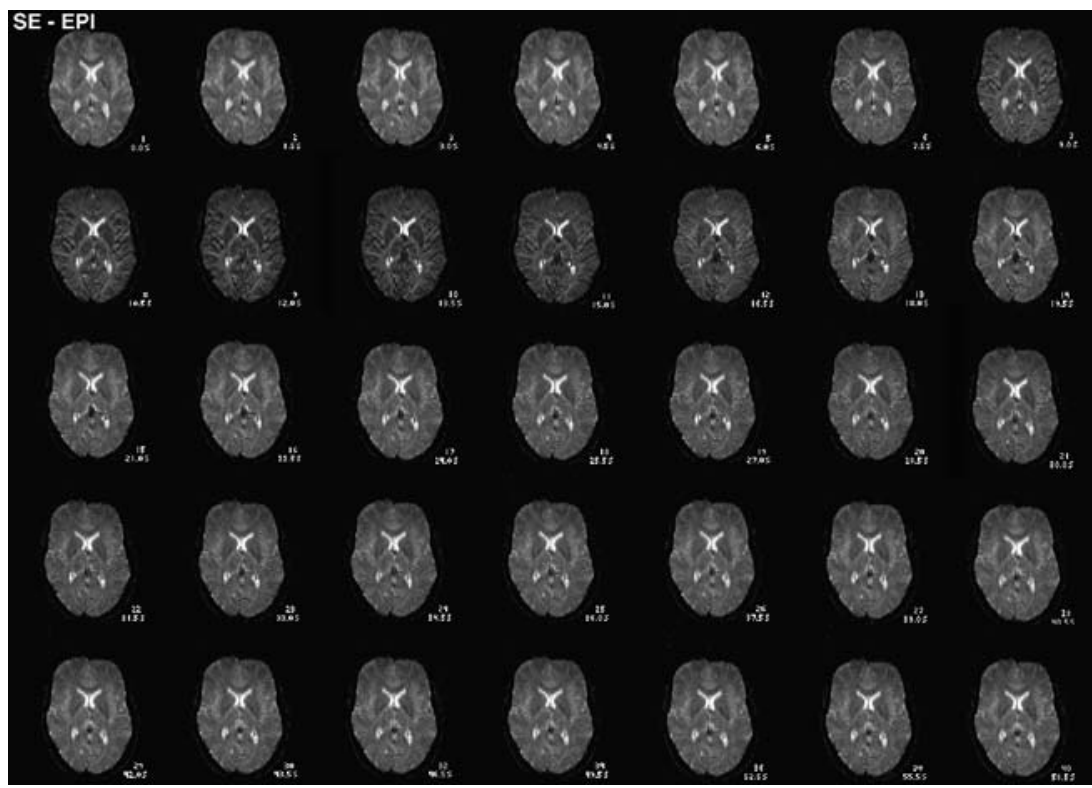

teractions; magnetic susceptibility phenomena extend outside of the vessels for a distance roughly equal to the radius of the blood vessels. This means that these effects are not just limited to the blood pool but involve the entire brain; more spins are affected by the passage of paramagnetic contrast agents. By affecting more spins, a bigger signal change is achieved. The improved signal results in more reliable PWI maps. In addition, the haemodynamic information supplied by magnetic susceptibility PWI is more tissue perfusion weighted than T1-weighted dipole-dipole imaging.

\section{Basic principles of dynamic magnetic susceptibility imaging}

Dynamic magnetic susceptibility imaging relies on the rapid acquisition of as many images as possible during the passage of contrast media through the brain to measure the degree of $\mathrm{T} 2 / \mathrm{T} 2 *$ signal changes over time (Figs. 1, 2, 3). Rapid and well-timed imaging is necessary because magnetic susceptibility effects rely on high concentrations of $\mathrm{Gd}$, which are typically present during the first passage of contrast agents through the capillary 


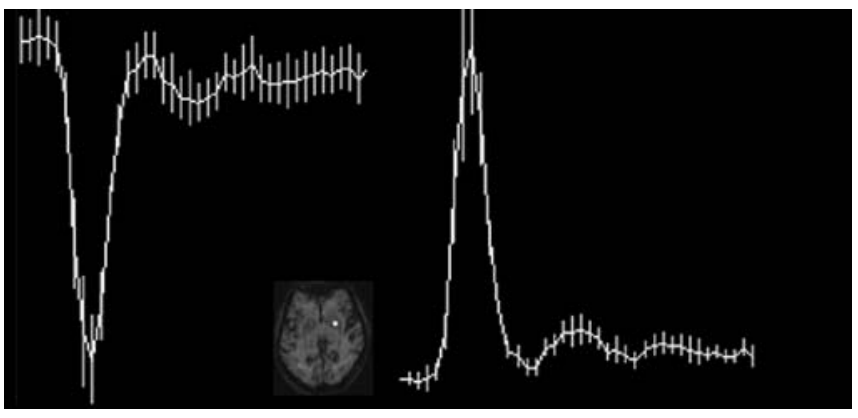

Fig. 3 Normal signal-vs-time graph in a voxel within the central grey matter. Each data point represents the signal intensity measured within the voxel in time. Error bars indicate the standard deviation. The curve is characterized by a baseline region that is ended by the arrival point of contrast agent with a sharp decline of the signal intensity. The signal drop recovers quickly after a peak signal change as the bolus washes out of the brain. A second, small signal drop results from the first bolus recirculation. The post-injection signal intensity remains somewhat lower compared with the initial baseline signal intensity due to residual, recirculating contrast agent. The signal-vs-time curve is then transformed into a concentration-vs-time curve or $\Delta \mathrm{R} 2$-vs-time curve by taking the negative $\log$ of the signal change. Cerebral blood volume $(\mathrm{CBV})$ is calculated from the area underneath the $\Delta \mathrm{R} 2$ curve. If the arterial input function is known, cerebral blood flow (CBF) maps can be calculated. Mean transit time (MTT) maps are calculated by dividing $\mathrm{CBV}$ by $\mathrm{CBF}$. Additional maps are created relying on different characteristics of this curve, e.g. time-to-peak (TTP) signal change or full width half maximum (FWHM)

bed. Recirculation phenomena rapidly dilute the passing Gd bolus. The measured MR signal intensity change vs time curve is converted into a contrast agent tissue concentration vs time curve (Fig. 3). Both empirical and theoretical studies have shown that the degree of signal drop in a voxel is directly related to the amount of contrast agent in that voxel $[9,11]$. This relationship between the measured signal change over time and the concentration of contrast agent in a voxel over time is the key link that allows calculations of cerebral haemodynamics. This non-invasive technique for measuring cerebral perfusion was first used by Villringer et al. in 1988 [12]. The mathematical algorithms used to calculate the haemodynamic data are similar to the indicator or tracer dilution methods developed at the end of the nineteenth century $[13,14]$. PWI uses a simple extension of this principle: if one compares the signal intensity of voxels before and after the injection of a known amount of contrast agent, the signal change can only be due to the addition of the contrast agent. Because the signal drop is directly related to the concentration of the $\mathrm{Gd}$ in the blood pool, CBV can be calculated by knowing the amount of Gd that was injected.
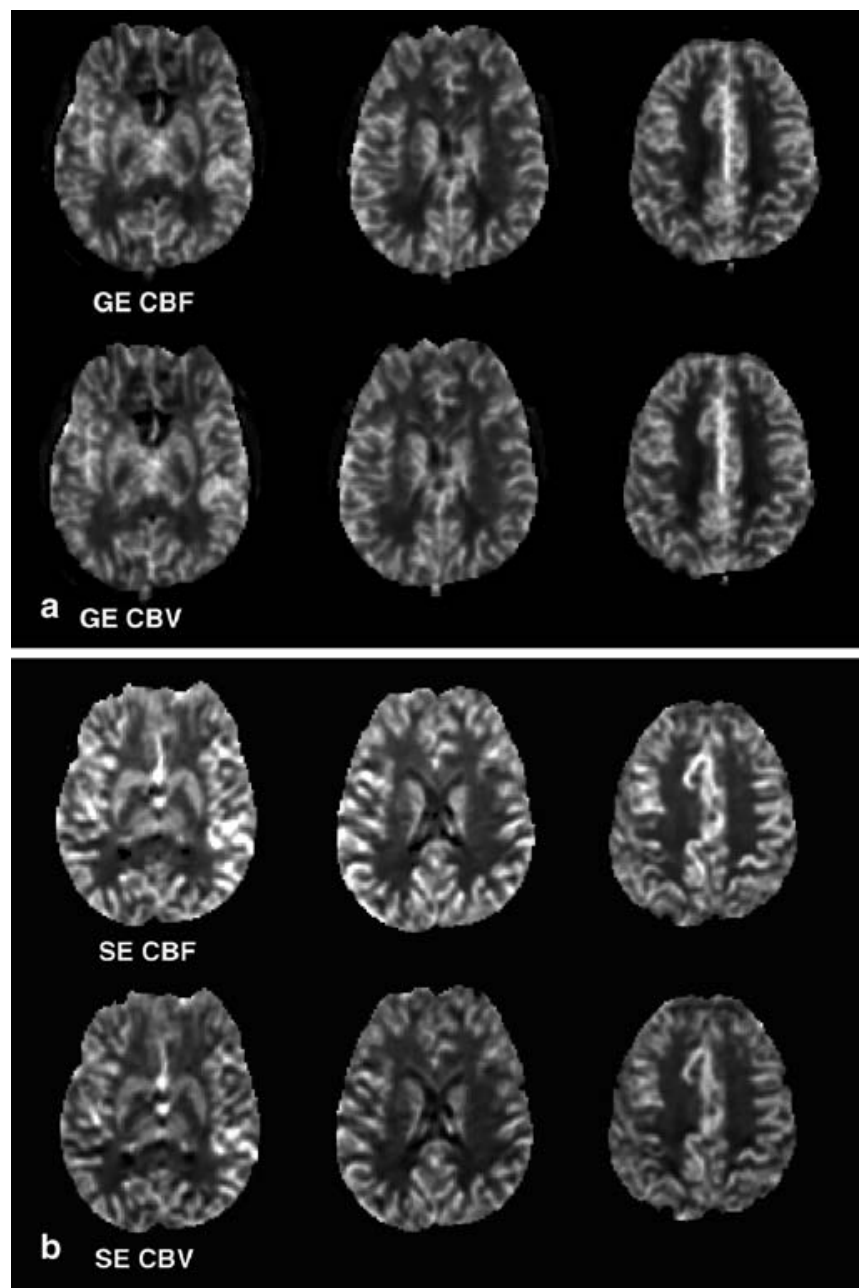

Fig. 4a, b The CBF and CBV maps in a 16-year-old boy. The maps are calculated on a voxel-by-voxel basis from a GE EPI sequence and an SE EPI sequence. Both sequences show a good discrimination between grey and white matter. In both maps the $\mathrm{CBF}$ and $\mathrm{CBV}$ of the grey matter is higher than that of the white matter. In the normal brain the $\mathrm{CBF}$ image appears similar to the CBV image

\section{MR sequences in dynamic susceptibility contrast imaging}

The primary goal of susceptibility MRI is to generate a T2/T2* signal vs time graph by measuring a maximum of data points during the first passage of the contrast agent. Echo-planar (EPI) sequences are typically used because the short acquisition time $(<100 \mathrm{~ms})$ per slice allows acquiring a large number of images per time period at multiple locations.

Echo-planar techniques can be combined both with T2-weighted spin-echo (SE) and T2*-weighted gradientecho (GE) sequences (Figs. 1, 2). The SE sequences have been proven to be more advantageous than GE se- 

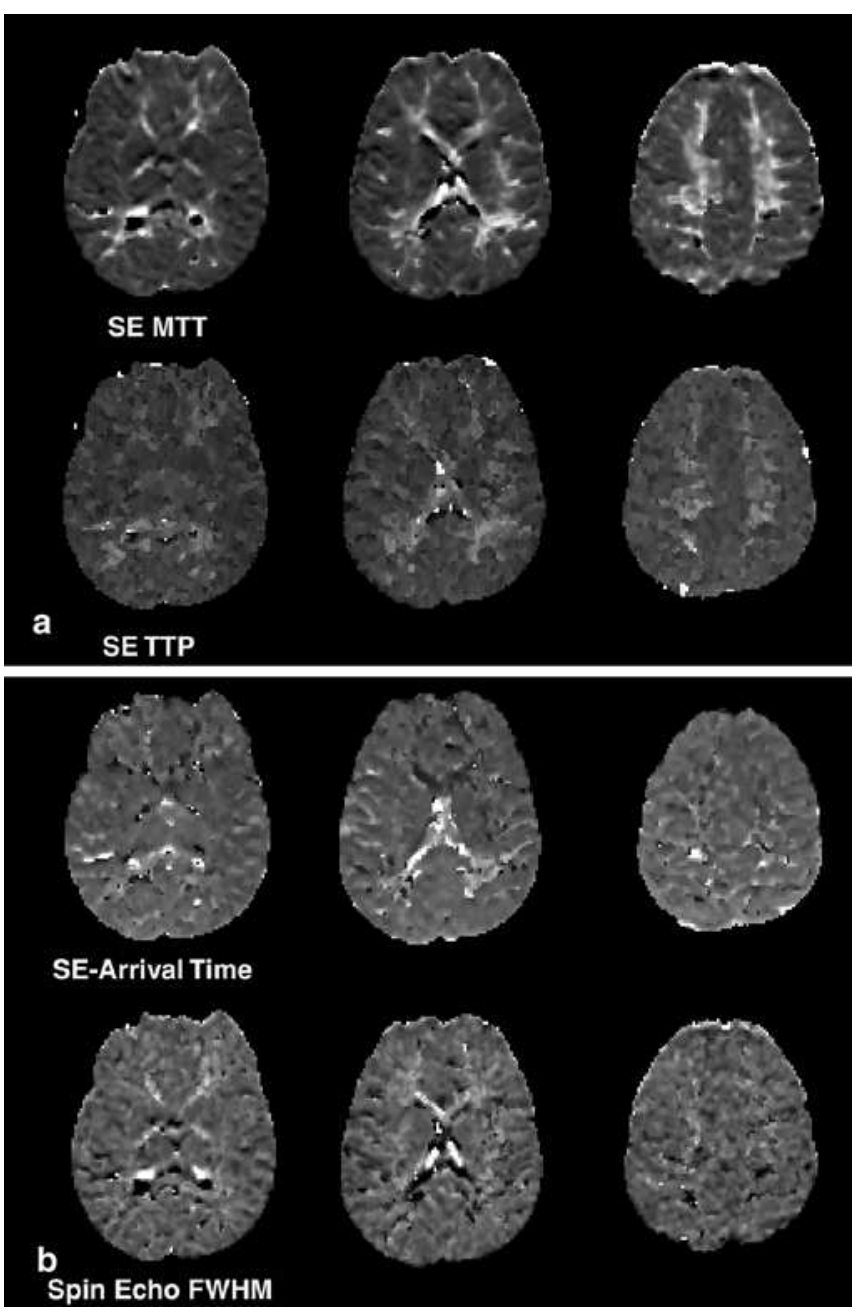

Fig. 5a, b The MTT, TTP, bolus arrival time and FWHM maps in a 16-year-old boy. These maps are calculated from different characteristics from the $\Delta \mathrm{R} 2$-vs-time curve. The MTT and TTP maps are most frequently used next to CBF and CBV maps. The advantage of these maps is their uniform signal intensity. Small changes are easily identified, which can go undetected on CBF and CBV maps

quences because of their higher sensitivity for the microvascular perfusion $[11,15]$. The microvascular image weighting brings PWI into a biologically relevant range. Subtle differences between the blood volume of grey and white matter ( 4 vs $2 \%$ blood volume) can consequently be resolved (Figs. 4, 5, 6). The increased specificity for the microvascular perfusion is, however, accompanied by a small decrease in the signal-to-noise ratio (SNR). Increasing doses of Gd can compensate for the SNR reduction.

In clinical practice the patient is examined in a standard head coil with a sequential multislice single-shot SE or GE EPI acquisition. Images are typically obtained for $1 \mathrm{~min}$ to include precontrast baseline images, to cap-

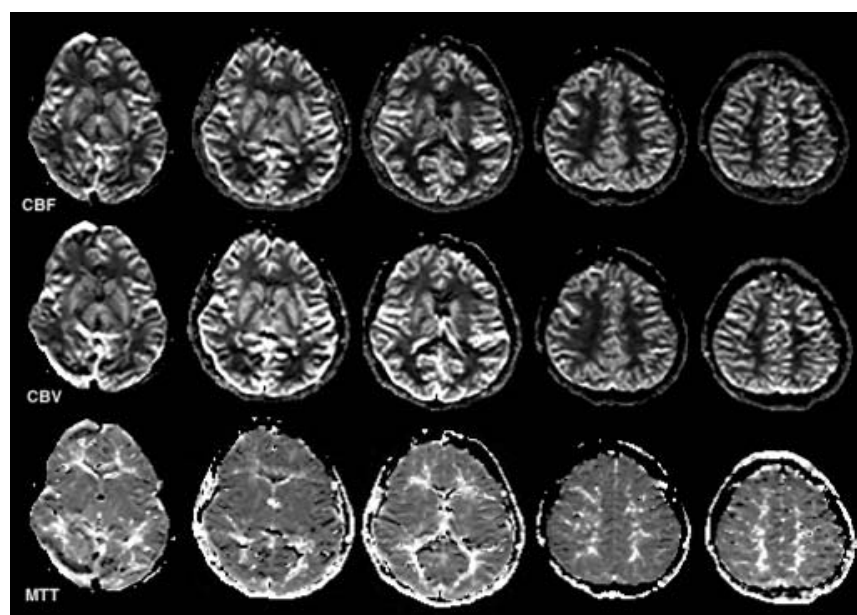

Fig. 6 The CBF, CBV and MTT maps in a healthy 8-year-old child. The CBF and CBV is larger in the grey matter than in the white matter. The internal capsule is easily differentiated from the adjacent basal ganglia. The cortex is well perfused. The CBF and CBV maps should be symmetrical and identical

ture the first passage of the contrast agent and the postinjection phase which includes the second signal drop due to the recirculation of the contrast bolus as well as the slow recovery of the signal to baseline due to the progressing dilution of the contrast agent (Fig. 3). Since the $\mathrm{CBV}$ is calculated on the basis of the signal drop relative to the precontrast baseline, an adequate estimation of the baseline (signal) is essential. A reliable baseline signal is best defined by as many images as possible. Depending on the MRI hardware, each slice is measured up to 80 times. The first passage is usually visible on 10-12 images (Fig. 3). One simulation study found that 50 or more images (per slice) increase the SNR substantially [16]. The number of slices usually ranges between 5 and 20.

Depending on the performance of the MR gradient systems, the number of slices is limited by the repetition time (TR). By increasing the number of slices, the TR will inevitably increase. Long TRs are, however, disadvantageous because the SNR will drop and secondly the passage of the contrast bolus is sampled by fewer images. On the other hand, to avoid T1-weighting, the TR should not be too short (e.g. TR $<500 \mathrm{~ms}$ ). Currently, a TR of $1500 \mathrm{~ms}$ is thought to be a good compromise. The echo time (TE) should be a balance between an adequate T2-weighting and sufficient T2 signal. Currently, a TE is advised between 60 and $75 \mathrm{~ms}$. The slice thickness may vary between 5 and $10 \mathrm{~mm}$, the field of view between 200 and $240 \mathrm{~mm}$ and the acquisition matrix between $128 \times 128$ and $256 \times 256$. As with any parameter, the appropriate value is a balance or compromise between advantages and disadvantages. The parameter should be optimized for the available field strength, gradient per- 
formance, region of interest, suspected pathology, and patient characteristics (young child vs adult). In young children, for instance, the slice thickness should be reduced as much as possible (3-4 mm). In addition, the smaller brain size allows to reduce the field of view $(160-180 \mathrm{~mm})$, consequently increasing the spatial resolution. Currently, no age-specific TR and TE times have been determined or published.

High field strengths are desirable (1.5 $\mathrm{T}$ and higher) because the SNR will increase and consequently the haemodynamic maps will be more reliable. The increased SNR is especially favourable in young children.

Perfusion-weighted MRI should always be performed along with conventional MRI. The short acquisition times easily allow an integration of PWI in most routine departmental imaging protocols. PWI should, however, be performed before contrast-enhanced conventional MRI sequences are measured. Postcontrast T1-weighted images can usually follow PWI, as the acquisition times of PWI are short.

\section{Rationale on magnetic susceptibility contrast agents}

Susceptibility effects can be induced by using intrinsic or extrinsic contrast agents. Intrinsic contrast agents are used in functional MRI to examine brain function [17, 18]. Intrinsic contrast agents can also be used in PWI (e.g. ASL). A major advantage is that no contrast agents have to be injected and that the measurements can be repeated as often as necessary within the same examination. A major limitation is that the signal changes are small (for ASL: of the order of $1-2 \%$ at $1.5 \mathrm{~T}$ ).

The rationale of using an extrinsic contrast agent is to take advantage of the much larger susceptibility effects of $\mathrm{Gd}$. The injection of a single dose of $\mathrm{Gd}(0.1 \mathrm{mmol} / \mathrm{kg}$ body weight) results in an average signal drop of $10-15 \%$ at $1.5 \mathrm{~T}$ (SE EPI sequences; Fig. 3). The signal drop can even be enhanced to $20-30 \%$ by increasing the dose to $0.2 \mathrm{mmol} / \mathrm{kg}$ (Fig. 7). This greater signal drop will increase the discrimination between grey and white matter and will show subtle changes of the cerebral haemodynamics in early stages of vascular disease. There is, however, also an upper limit. If the signal decrease is greater than $40-50 \%$, additional Gd will decrease the quality of the perfusion maps [16].

Magnetic susceptibility contrast agents should be injected as a narrow bolus by a power injector for two reasons. First of all, T2-weighted magnetic susceptibility effects dominate image contrast only if high intravascular concentrations of Gd are achieved. Secondly, CBF is calculated from the shape and size of the peak signal drop vs time curve. A wide bolus might blur slight differences in arrival time between voxels. By using a bolus as narrow as possible, the information extracted from the sig-

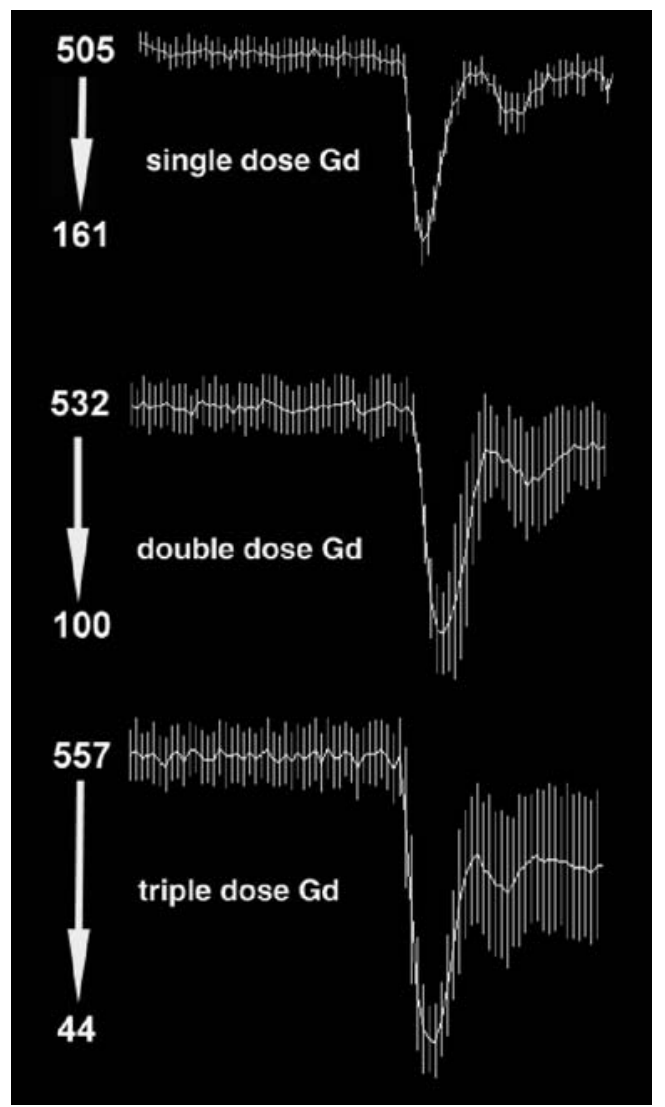

Fig. 7 Signal-vs-time graphs for different doses of gadolinium. The larger doses of contrast agent provide a greater signal change; greater signal changes typically increase the quality of the perfusion maps. Note that the numbers along the $y$-axis are arbitrary units displaying the change (loss) in signal intensity during the passage of the gadolinium

nal-vs-time curve can be more accurately translated in a CBF map. Methods to narrow the bolus of contrast agent include the use of a power injector with high injection rates $(5 \mathrm{ml} / \mathrm{s})$ and the use of more concentrated contrast agents or contrast agents with higher magnetic susceptibility effects (e.g. Dysprosium-based agents). In addition, a fully automated contrast injector allows flushing the system with a bolus of saline after the Gd injection. This will advance the remaining Gd within the tubes and within the patient's venous system towards the heart. The use of power injections is not yet established in very young children. Limited experience is currently available; most paediatric neuroradiologists favour a manual injection. The smaller volume of contrast to be injected in young children and the higher heart rate and faster circulation time are advantageous in children because they narrow the contrast bolus. This will increases the reliability of the haemodynamic maps.

Dynamic susceptibility contrast imaging relies on an intact blood-brain barrier (BBB) to keep the contrast 
agent intravascularly compartmentalized. In disease processes where the BBB is disrupted, the contrast agent leaks into the brain tissue. The decrease of the compartmentalization reduces local field inhomogeneities. In addition, the extravascular accumulation of contrast agents causes a local T1 shortening. Both phenomena counteract T2-signal decreases from susceptibility effects. Postprocessing algorithms can compensate for moderate degrees of BBB leakage [11]. Alternatively, a pre-dose of 0.0025 or $0.005 \mathrm{mmol} / \mathrm{kg}$ of $\mathrm{Gd}$ can be given $5 \mathrm{~min}$ before the bolus injection. In areas of a disrupted BBB, the Gd will shorten the T1 relaxation. By using a 5-min delay, the baseline images will already incorporate the signal change due to the $\mathrm{T} 1$ shortening. The passing bolus of Gd will subsequently reduce the signal proportionally to T2 susceptibility effects. The predose of Gd compensates for the T1 shortening effects of the leaking Gd, but cannot, however, overcome the decrease in compartmentalization. Finally, the use of Dysprosium chelates, which display less $\mathrm{T} 1$ relaxivity effects while the $\mathrm{T} 2$ magnetic susceptibility effects are more pronounced, partially compensate for a leaking BBB [19].

\section{Computation of haemodynamic maps}

The measured T2 signal intensity-time curve is the starting point of all haemodynamic map calculations (CBV, CBF, MTT). This curve is available for each voxel within the imaging plane (Fig. 3). The MR signal intensity curve is converted into a change in $1 / \mathrm{T} 2$, or $\Delta \mathrm{R} 2$-vs-time curve (Fig. 3). Because $\Delta \mathrm{R} 2$ and the tissue contrast agent (e.g. Gd) concentration show a linear relationship $\left(\Delta \mathrm{R} 2=\mathrm{k}_{2}[\mathrm{Gd}]\right)$, this curve equals a contrast-agent-vstime curve. $\left(\mathrm{k}_{2}\right.$ is a constant that depends on tissue magnetic field strength, and MR pulse sequence; $[\mathrm{Gd}]$ is the tissue concentration of gadolinium.)

Maps of CBV (Fig. 4) are calculated by integrating the area under the $\Delta \mathrm{R} 2$ curve or concentration-time curve over time for each pixel. The CBV parameters are no absolute values but represent relative scalars because the proportional constant $\mathrm{k}_{2}$ between $\Delta \mathrm{R} 2$ and absolute contrast agent concentration cannot be determined without sampling the blood.

The calculation of CBF maps (Fig. 4) require an additional step. An arterial input function (AIF) has to be specified which provides information about the timing of the contrast agent. Typically, another tissue-vs-time curve within voxels next to the major artery that feeds the tissue of interest is used as AIF (Fig. 8). In analogy to CBV, $\mathrm{CBF}$ values are relative rather than absolute. Maps of the mean transit time are rendered by dividing $\mathrm{CBV}$ into $\mathrm{CBF}$ (Fig. 5). A number of other "time" maps can be useful, depending on the pathology. These maps are usually simple to calculate and include maps of the arrival time of the bolus and the time to the peak signal change (TTP; Fig. 5).

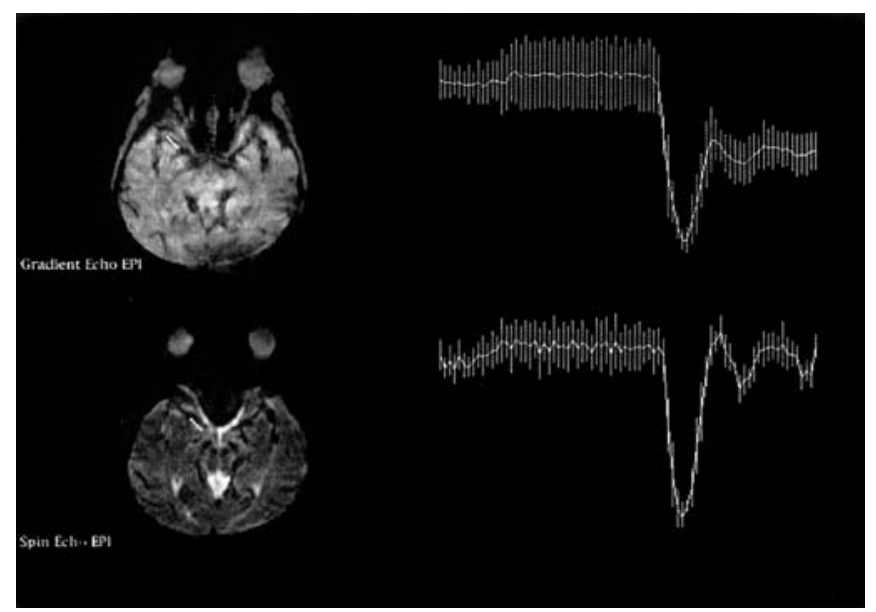

Fig. 8 Normal signal-vs-time graph in voxels along the right middle cerebral artery (MCA) for a GE EPI and SE EPI sequence. These curves are used as arterial input function to calculate CBF. The signal drop is larger for GE EPI sequences compared with SE EPI sequences

All haemodynamic parameters are calculated on a voxel-by-voxel basis and can be displayed as a greyscale map (Figs. 4, 5, 6). The CBV and CBF maps show a cortico-medullary differentiation due to the differences in CBV and CBF between grey and white matter. The MTT maps can be helpful as lesion conspicuity is increased due to the more uniform appearance.

There are a number of potential difficulties related to the AIF of which one should be aware. The AIF can be influenced by variations in injection conditions and patient physiology. Delays and dispersion of the contrast bolus that occur during the passage of the bolus from the site of AIF measurement to the tissue of interest as well as partialvolume effects at the site of AIF measurement can introduce an error in the calculation of CBF. Furthermore, the tracer kinetic model assumptions of perfusion that are used for normal perfused brain tissue may be invalid in cerebral ischaemia. Consequently, perfusion maps should always be encountered with caution, in particular when absolute quantification is attempted. The reliability of absolute quantifications is determined by the quality of the AIF. Deconvolution methods are currently under investigation to circumvent these limitations to provide reliable absolute quantifications $[20,21]$. PWI is, however, very effective in the identification of regions with abnormal perfusion.

\section{Arterial spin labelling}

Arterial spin labelling (ASL) generates absolute haemodynamic parameter by labelling or tagging arterial spins. Inflowing blood water magnetization is inverted or saturated by a radio-frequency pulse before entering the brain slice of interest. After waiting an appropriate time 

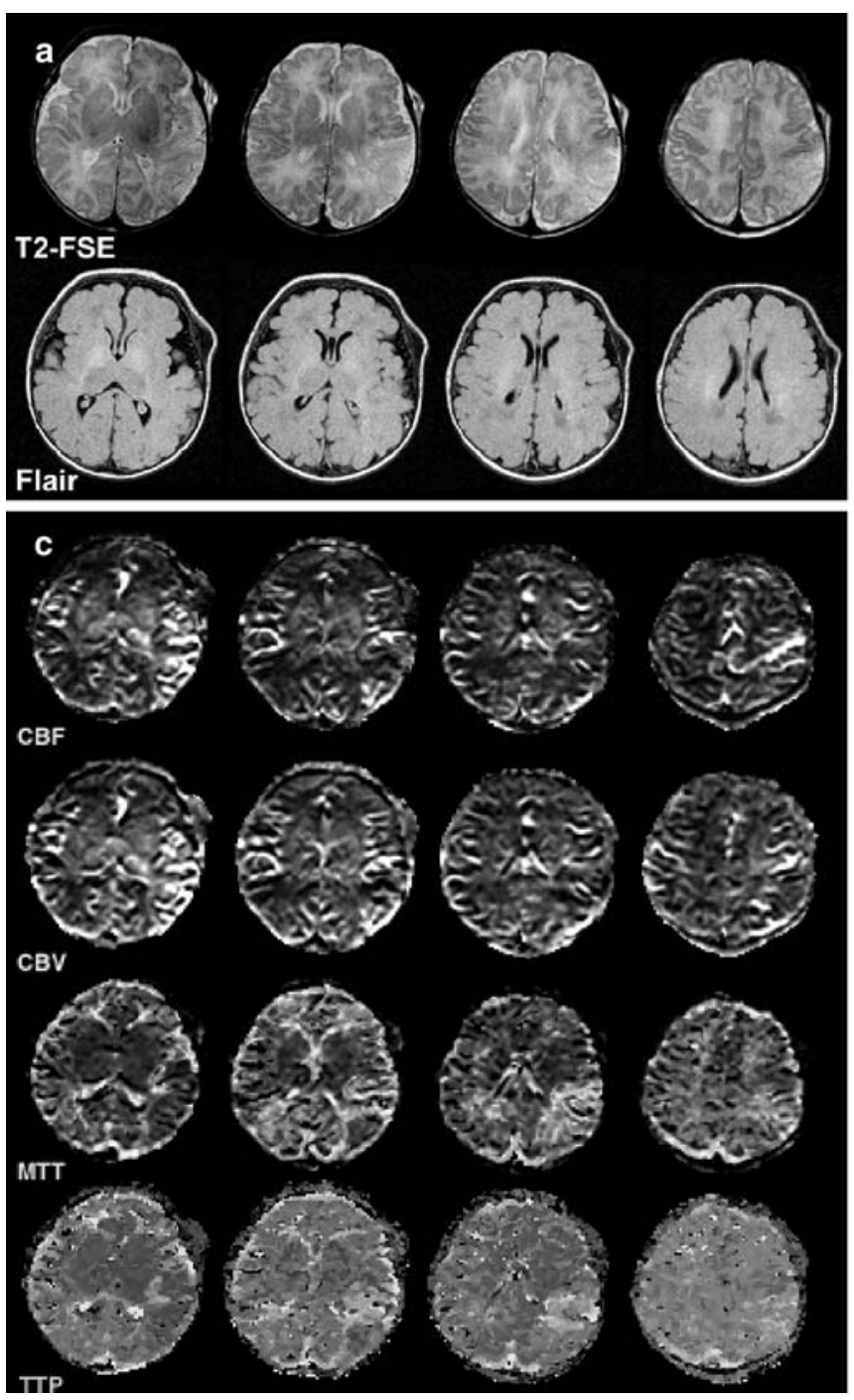

Fig. 9a-c A 1-day-old male (39 weeks of gestation) with acute neonatal stroke. The child presented with tonic clonic seizures at $24 \mathrm{~h}$ of age. a An extensive, wedge-shaped left MCA stroke is seen on T2-weighted fast-spin-echo (FSE) images. The lesion is barely seen on the fluid-attenuated inversion recovery (Flair) sequence. b The lesion is markedly hyperintense on isotropic diffusion-weighted images $(D W I)$ and hypointense on the apparent diffusion coefficient $(A D C)$ maps indicating acute neonatal stroke with decreased diffusion. The left posterolateral thalamus is also involved. c The perfusion maps show a decreased CBF, slightly increased CBV and a prolonged MTT/TTP matching the area of diffusion abnormality. No additional areas of perfusion abnormalities are encountered

interval, the labelled, inverted or saturated arterial blood spins enter the brain slice of interest and decrease the overall magnetization of this slice. The inflow of labelled spins generally produces a signal change of $1-2 \%$. The degree of signal change is directly related to the $\mathrm{CBV}$ and $\mathrm{CBF}[22,23]$.

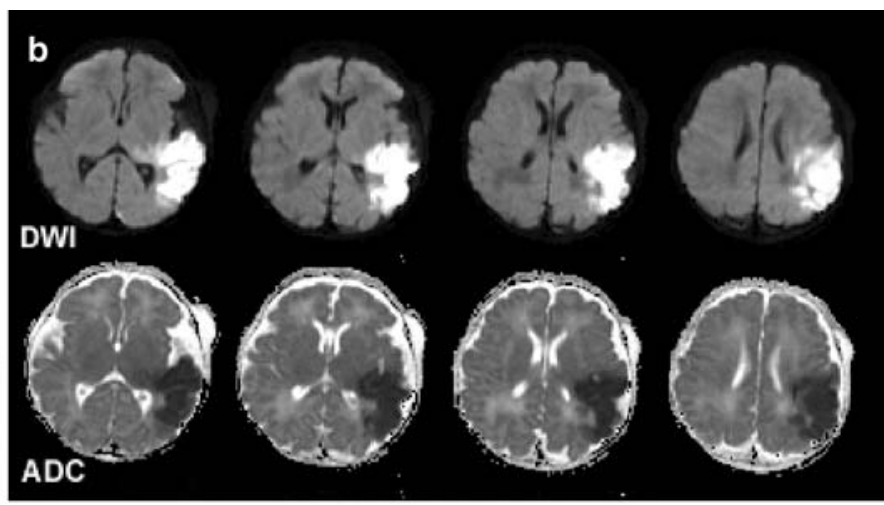

Arterial spin labelling techniques measure steadystate tracer levels by using the T1 magnetization state of the arterial spins themselves as a freely diffusible tracer. This has two advantages. First of all, steady-state methods allow multiple measurements or averaging periods to boost the SNR. Secondly, the free diffusion characteristics of the "tracer" give us in analogy to the magnetic susceptibility contrast techniques microvascular-weighted information. As soon as the labelled spins reach the brain slice of interest, the labelled spins diffuse into the extracellular space and interact with tissue spins or basically the tissue water.

The signal changes can be displayed by subtracting the image after spin labelling from a control image before spin labelling. The recorded signal changes are directly related to the $\mathrm{CBF}$ and $\mathrm{CBV}$. Consequently, absolute $\mathrm{CBV}$ and $\mathrm{CBF}$ parameters are calculated. Additional advantages of ASL are the insensitivity to susceptibility artefacts which enhances the reliability of measurements near the skull base and the possibility to selectively label arteries by unilateral or localized labelling coils. This allows examination of different vascular territories independently which can be used to evaluate the functional efficiency of collateral vascular supplies. A major disadvantage is the low signal change $(1-2 \%)$ and consequently the low SNR that requires long acquisition times (up to $10 \mathrm{~min}$ ), making the technique susceptible for motion artefacts. This can be especially troublesome in children. Only few reports are available on the use of ASL in children [24].

\section{Perfusion-weighted imaging in children}

Cerebrovascular disease (CVD) in children includes primary (e.g. acute embolic stroke, primary vasculopathies, arteriovenous malformations) and secondary vascular disorders (e.g. sickle cell disease, metabolic diseases, collagen vascular disorders, inflammatory/para-inflammatory conditions). In secondary disorders, the cerebrovascular system is not the primary site of pathology but 
Fig. 10a-c A 19-month-old boy with severe anoxic brain injury due to prolonged cardiac arrest. a Conventional MRI shows a discrete T2-hyperintensity within the posterior aspects of both lentiform nuclei and thalami. b The lesions are DWI hyperintense with associated ADC hypointensity indicating decreased diffusion. c Haemodynamic imaging reveals an increased CBV and CBF (MTT consequently unchanged) related to post-ischaemic hyperaemia
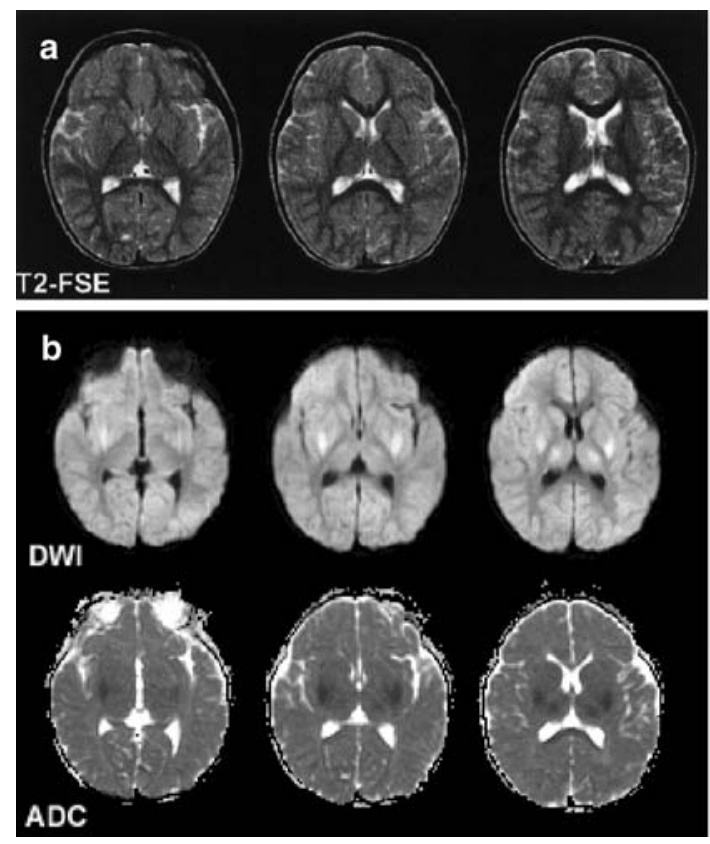

gets affected during the disease course. Secondary CVD in children tend to be more often thrombotic than embolic [25].

Because CVD is more prevalent in children than previously thought, [25] and represents a significant source of morbidity and mortality [25], the need for a minimally invasive and reliable haemodynamic imaging tool is obvious. The non-invasive character of PWI, the susceptibility for microvascular haemodynamic alterations, the short acquisition times, the lack of ionizing radiation and the presently widespread availability of MRI scanners make PWI ideally suited for children. In addition, the faster heart rate, the smaller total blood volume and the smaller size of the brain in children are advantageous. PWI can easily be added to conventional MRI integrating conventional-anatomical and functional-haemodynamic data (Figs. 9, 10, 11, 12, 13).

The indications for PWI in children are similar to those in adults. Most experience has thus far been collected for cerebrovascular occlusive diseases (cerebral ischaemia) and for intracranial neoplasm (e.g. tumour grading, differentiation between tumour recurrence and radiation necrosis). Other potential applications include hypoxic ischaemic encephalopathy or perinatal asphyxia, near drowning, intoxications, metabolic diseases, head trauma, migraine, epilepsy and any kind of cerebral disease that is accompanied by changes in the cerebral haemodynamics.

Perfusion-weighted MRI studies can possibly provide more insight into the understanding of the normal and pathological development and maturation of the brain. Furthermore, PWI could give important data about tu- mour angiogenesis allowing a better understanding of tumour growth and the presumed relation between perfusion and malignancy grade. PWI can also be used to guide and monitor treatment.

\section{Developmental changes of cerebral haemodynamics in children}

Knowledge about the normal pattern of brain perfusion as well as normal values of brain perfusion in the developing brain are essential for interpreting disease-related changes in cerebral perfusion. Because the child's brain is subject to a continuous maturation, perfusion values are expected to change during the progressing myelination/maturation. Currently, limited PWI results are available; most information has been extracted from PET and SPECT studies [26, 27].

Tokumaru et al. [26] studied the 123I-IMP uptake in the developing brain by SPECT. Their study showed that changes in ${ }^{123}$ I-IMP uptake parallel both temporally and topographically the changes in $\mathrm{T} 1$ and $\mathrm{T} 2$ relaxation times due to progressing myelination confirming the tight relation between cerebral perfusion and myelination as postulated by von Monakow at the beginning of the past century [28].

The change in the regional distribution and magnitude of perfusion in the maturing brain likely reflects the anatomic evolution of the cerebral vasculature. This process appears to be markedly influenced by the brain's functional and metabolic status. As neurons or neuronal centers approach maturity, they recruit an increasingly rich 


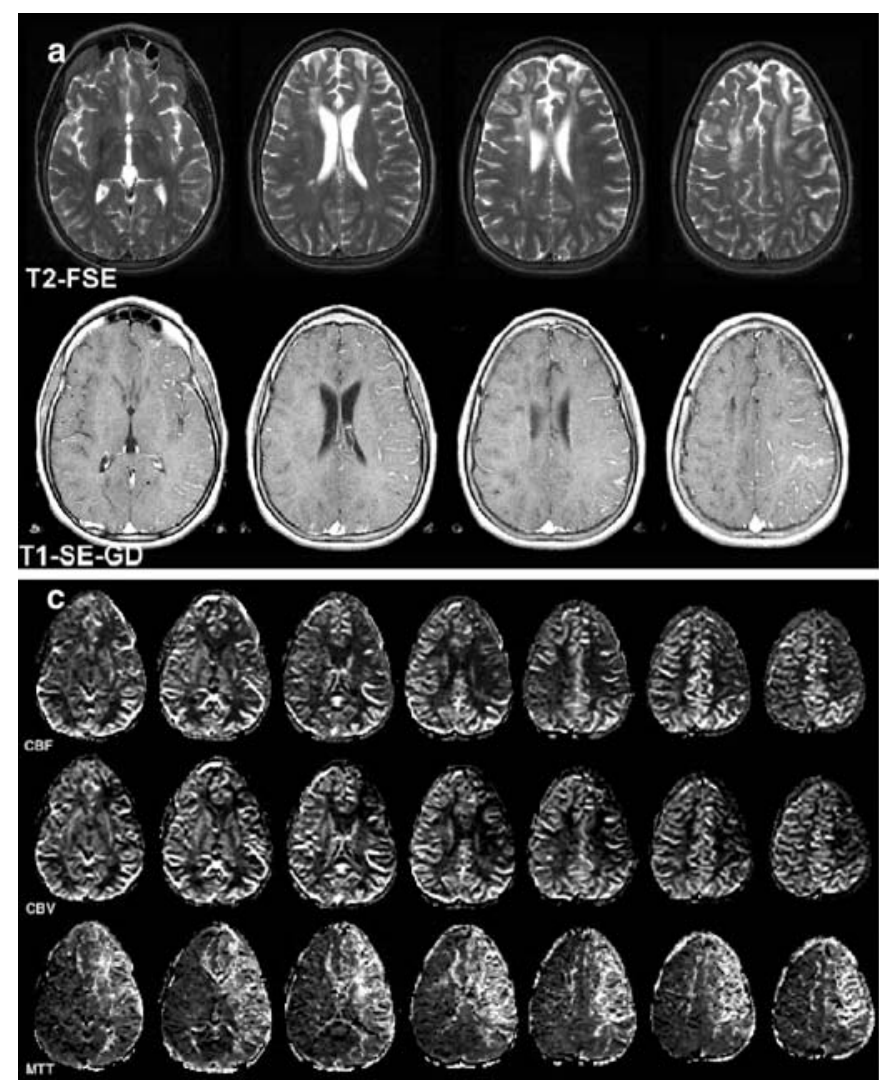

Fig. 11a-d A 12-year-old boy with sickle cell disease. On the day of MRI a third stroke event occurred with mild right-sided weakness and severe language defects. a Conventional MRI including T2 FSE and contrast-enhanced T1 SE imaging showed a discrete T2 FSE hyperintensity within the left frontal, parietal and insular cortical regions as well as a discrete leptomeningeal, sulcal enhancement. b The DWI shows multiple hyperintense, ischaemic lesions with decreased diffusion in the previously mentioned locations. $\mathbf{c}$ The CBF and CBV maps reveal a large area of hypoperfusion involving the left ACA and MCA territories. The MTT is markedly prolonged. The mismatch between DWI and PWI abnormality indicates tissue at risk for future infarction. d Follow-up imaging several months later showed a new, subacute T2 FSE hyperintense ischaemia within the area of initial hypoperfusion despite several blood transfusions

capillary network [29]. High blood flow is seen in myelinating and myelinated areas of the brain during the neonatal period that may reflect the increased energy demands of the biosynthetic processes associated with progressing myelination $[30,31]$. Blood flow to the white matter has been shown to be much higher during maturation than at completed maturation. These findings support the theory that progressing myelination increases the metabolic demand and consequently the CBF [30]. Another explanation is that myelination, $\mathrm{CBF}$ and cerebral metabolic rates are all related to increasing activity in specific regions of the brain that are functionally important during specific developmental periods [26]. Takahashi et al. [27]
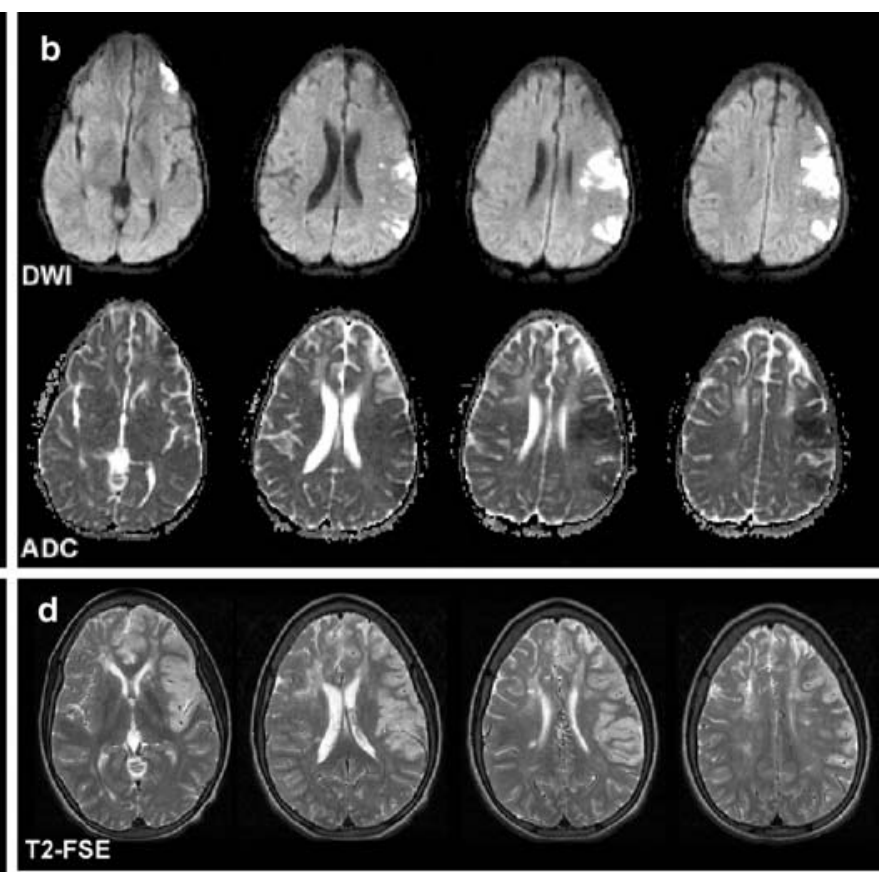

found that $\mathrm{CBF}$ is lower in the neonatal period than in older children and adults, increases significantly during early childhood with a peak around 7 years and finally gradually reaches adult values during adolescence. They also showed age-dependent regional differences in cerebral perfusion. The last area in which the cerebral perfusion increases appeared to be the frontal association cortex, whereas the cerebral perfusion was prominent in the occipital lobe in every age group. The dynamic changes in cerebral perfusion parallel the physiological developmental state within the different brain areas.

Ball and Holland [25] evaluated more than 1000 PWI examinations performed in children between 6 days and 18 years. The normal grey-to-white-matter CBV ratio ranged between 3 and 4:1. Higher ratios were seen in children under 2-3 years of age. In healthy children, $\mathrm{CBV}, \mathrm{CBF}$ and MTT maps should always be symmetrical for both hemispheres (Fig. 6).

\section{PWI in cerebral ischaemia}

Perfusion-weighted MRI has proved its value in acute cerebral ischaemia [6]. Diffusion-weighted MRI (DWI) identifies tissue injury (cytotoxic oedema) within minutes after ischaemia [32]. PWI in combination with DWI can detect critical cerebral hypoperfusion before neuronal cell injury becomes irreversible and can identify tis- 


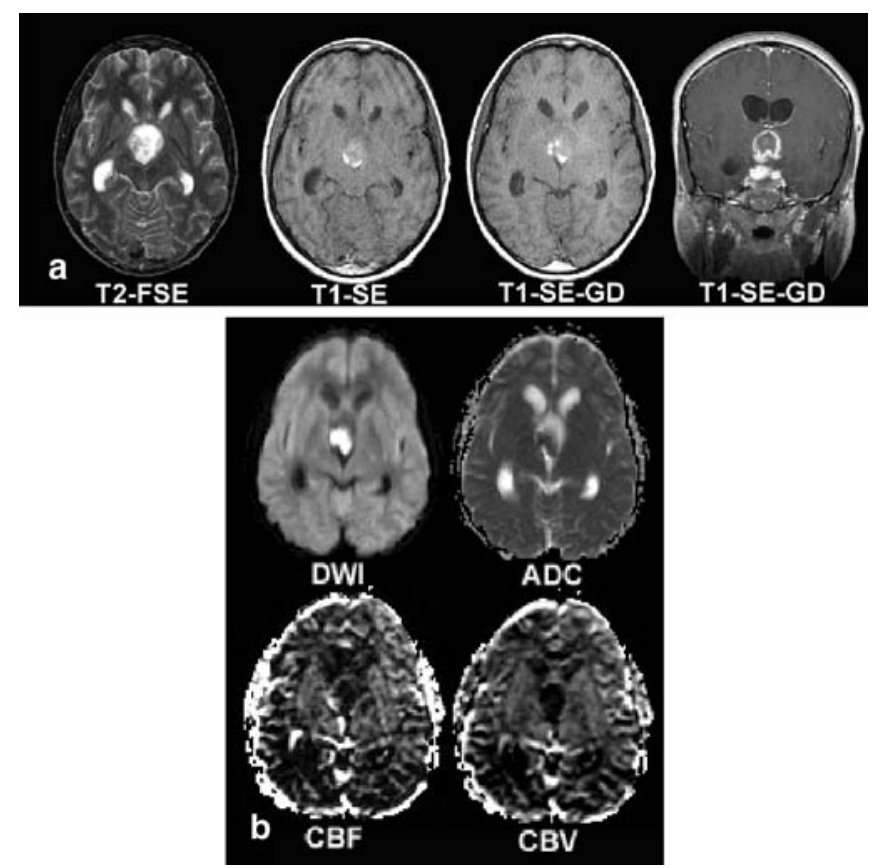

Fig. 12a, b An 11-year-old girl with a low-grade astrocytoma. a Conventional MRI revealed a hypothalamic/chiasmatic astrocytoma. The lesion is slightly T1 hyperintense due to an intra-lesional haemorrhage. b The lesion is both DWI and ADC hyperintense with a hypointense peripheral ring that results from susceptibility effects related to the intra-lesional haemorrhage. The low $\mathrm{CBV}$ and CBF map values are consistent with the low malignancy grade of the tumour. The haemodynamic maps have been corrected for a possible leaky blood-brain barrier

sue at risk for future infarction. The combination of DWI and PWI allows discrimination between the core of infarction and the ischaemic penumbra. The core most probably represents irreversibly injured tissue, whereas the penumbra is believed to represent ischaemic but viable brain tissue [33]. Schlaug et al. [34] hypothesized that the ischaemic penumbra can be defined operationally by correlating the area of initial diffusion abnormality with the area of hypoperfusion on PWI. Matching areas of DWI and PWI abnormalities are considered as a possible imaging correlate to the core of the infarction, whereas a DWI/PWI mismatch (PWI $>$ DWI) is considered to correlate with the ischaemic penumbra (Figs. 9, 10, 11) [34]. Major advantages are that treatment can be started before tissue injury is irreversible, infarct progression can be limited, and possible preventive treatments for tissue at risk for infarction can be considered.

Perfusion-weighted MRI can also be of importance to identify spontaneous reperfusion [6]. Reperfusion can save ischaemic but viable, temporarily hypoperfused brain tissue. Reperfusion is, however, also thought to be associated with secondary injury to the brain [35]. Socalled reperfusion injury with decoupling of CBV, CBF and metabolic rate can be the target of neuroprotective treatments. Reperfusion injury is believed to be related to secondary injury in perinatal asphyxia [35].

One of the most important contributions of PWI in the management of children with acute neurological deficits is to rule out cerebral ischaemia. In most cases of acute neurological symptoms, cerebral ischaemia is included in the differential diagnosis despite the fact that many other diseases can present with acute deficits. Because conventional MRI can be misleading in the early stages of ischaemia and a possible delay in starting treatment can have far-reaching consequences, the exclusion of acute cerebral ischaemia by PWI/DWI is as valuable as the identification of cerebral ischaemia.

Finally, PWI may also be helpful in differentiating venous from arterial infarction. In venous infarction, an arterial deficit may be absent, whereas in arterial occlusion an arterial deficit is always present. In addition, the signal recovery due to the passage of Gd is markedly delayed in venous infarction, whereas this delay can be absent in arterial infarction [25, 36].

\section{PWI in primary or secondary cerebral vasculopathies}

Moya-Moya disease is one of the most frequent and bestknown primary cerebral vasculopathy in children with chronic progressive, large vessel obstructive disease. Secondary vasculopathies in children include homozygous sickle cell disease (Fig. 11), idiopathic vasculitis, secondary vasculitis and various metabolic diseases.

Perfusion-weighted MRI is very important in evaluating the cerebral haemodynamics especially concerning possible collateral circulations. PWI may reveal the collateral perfusion, give information about the significance of this collateral supply and possibly predict the likelihood of the haemodynamic success of a bypass.

Perfusion-weighted MRI has been proven to be useful in the diagnosis and follow-up of children with MoyaMoya disease [37, 38, 39]. In the initial presentation, an increased flow in the basal ganglia next to perfusion deficits and altered transit times within the cortex are observed. In the late stadium, PWI is helpful in assessing the chronic hypoperfusion. In contrast to SPECT, PWI allows to determine if the remaining cerebral perfusion results from the great cerebral arteries or from collaterals. The reconstruction of perfusion maps, especially CBF maps, can be difficult. The progressive stenosis of the great cerebral arteries makes it difficult to find a reliable AIF.

\section{PWI in cerebral tumours}

Several studies in adult patients have linked tumour grade with changes in CBF. One study indicated that tumours with any regional $\mathrm{CBV}$ value over twice that of 

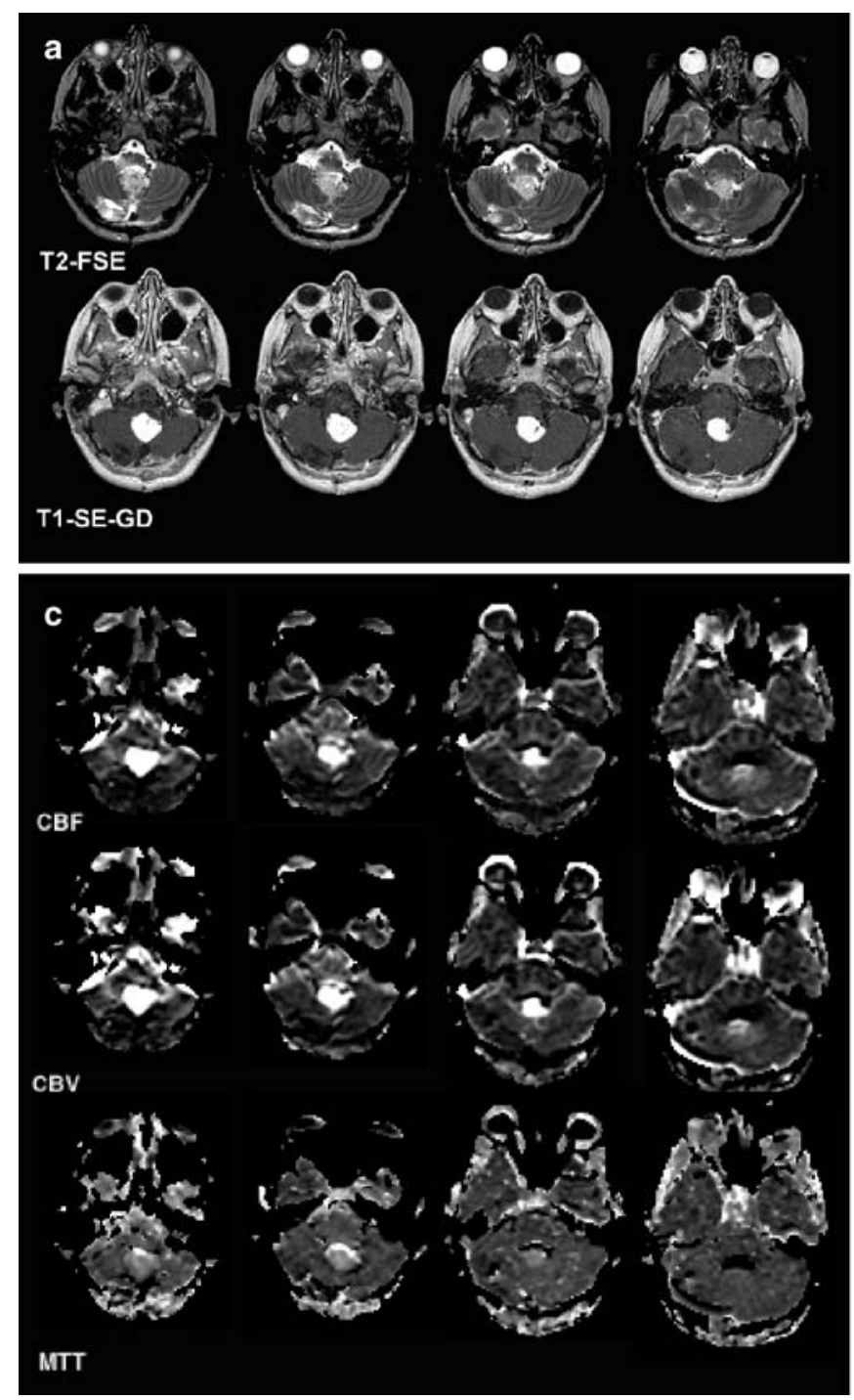

Fig. 13a-c A 16-year-old girl with a fourth ventricle haemangioblastoma. a Conventional MRI shows a T2-hyperintense, contrastenhancing midline tumour within the region of the foramen of Magendie. Multiple, enlarged tumour vessels within the tumour and within the adjacent cerebro-pontine cisterns are seen. b The tumour is DWI hypointense and ADC slightly hyperintense. c The $\mathrm{CBV}$ and $\mathrm{CBF}$ values are markedly increased due to the high vascularity of the tumour. The MTT is also increased

the white matter have a high likelihood of having highgrade components, whereas tumours with maximum $\mathrm{CBV}$ values less that of 1.5 times white matter are usually low grade [40]. Since maps of CBF have become available only recently, few studies have focused on correlating CBF with tumour grade. Initial results indicate a similar relation between $\mathrm{CBF}$ and tumour grade [41]. These links between tumour grade and cerebral haemodynamics could, however, not yet be reproduced for paediatric brain tumours (Figs. 12, 13). Ball and Holland re-

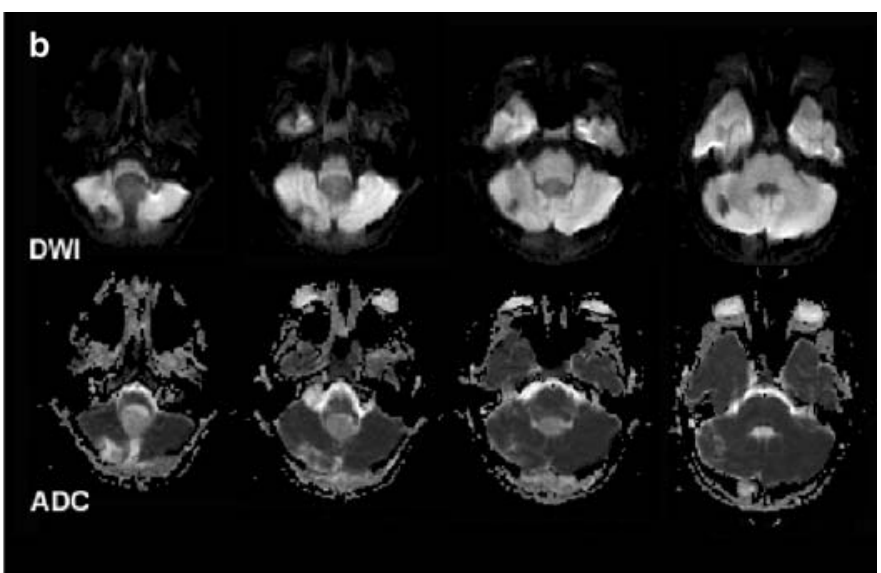

ported similar $\mathrm{CBF}$ values for pilocytic astrocytomas and medulloblastomas [25].

Most authors agree over the value of PWI in differentiating tumour recurrence from complications of radiation therapy or chemotherapy. Imaging findings can be indistinguishable on conventional MRI [42]. PWI can, however, distinguish between radiation necrosis and viable tumour. Tumour tissue will show normal or increased haemodynamic parameter (CBV), whereas radiation necrosis is characterized by decreased CBV values [43]. Similar reductions in CBV are encountered in tissue injury and necrosis related to chemotherapy [44].

Brain tumours often display different grades of malignancy in different areas of the tumour bulk. Correct tumour grading relies on the biopsy of the tumour components with the highest grade of malignancy. Haemodynamic maps can be helpful in directing biopsies to the areas of highest malignancy (increased CBV) [45]. Currently, no prospective studies have been published in paediatric brain tumours.

One of the most exciting fields of active research in haemodynamic imaging of brain tumours is focused on characterizing tumour angiogenesis. Angiogenesis refers to the ability of tumours to induce the proliferation of new blood vessels (neovascularization). Angiogenesis is believed to play an important role in the growth and spread of cancer. Once a malignant cell transformation has occurred, every increase in tumour cell population must be preceded by an increase in new capillaries converging to the tumour $[46,47]$.

Tumour vessels differ from vessels in normal tissue both qualitatively and quantitatively. Tumour vessels are usually disorderly and often large, dilated and tortuous with increased distances between the separate vessels. In addition, average tumour vessels have diameters two to three times that of normal tissue. Furthermore, during the initial formation of the tumour vascular bed, capillary sprouts and blind-ended microaneurysm-like saccular vessels are observed at the tumour margins. With the progress- 
ing evolution of the vascular bed and progressing tumour size, these buds anastomose to other new vessels with continuous increase of vessel size $[48,49]$. The microvascular architecture and the disorganization of neovascularity with multiple blind-ending vessels and other morphological changes are believed to be responsible for significant mismatches between CBF and CBV in tumours. These mismatches can represent a biomarker of disorganization and tumour malignancy. The CBF-CBV mismatch possibly correlates with how many angiogenic vessels are present, since many angiogenic vessels are blind ending.

Techniques which could serially evaluate angiogenesis non-invasively would be extremely valuable to better understand tumour growth, tumour expansion, and progressing tumour vascularization. In addition, the results from anti-angiogenic therapeutic agents or treatments could be monitored, thereby providing insight into why anti-angiogenic therapy succeeds or fails.

Few angiogenesis-specific imaging tools are currently available. Preliminary data suggest that the distribution of capillary size can be extracted from PWI techniques. Spin-echo PWI sequences show a significant degree of microvascular weighting compared with a more uniform vessel size dependence seen on GE PWI sequences. In other words, the degree of flow-related signal changes on SE EPI sequences depends on the vessel size, whereas signal changes on GE sequences are less dependent on the vessel size [50]. This difference in signal decay can be used to determine average capillary diameter in a voxel [51]. The ratio of the change in $\mathrm{T} 2 *$ and $\mathrm{T} 2$ relaxation rate increases nearly linearly with vessel size and therefore may provide an indication of the average vessel size within a voxel allowing to measure the degree of angiogenesis. This information can be achieved by interleaving a SE with a GE sequence during a single injection of contrast agent. Next to the possibility of mapping vessel size distribution, CBV and CBF can easily be measured by PWI. Consequently, PWI could serve as a promising technique to visualize tumour angiogenesis, and can consequently be used to monitor anti-angiogenic therapies in the future.

\section{References}

1. Roy CS, Sherrington CS (1890) J Physiol (Lond) 11:85-108

2. Grubb RL Jr, Raichle ME, Eichling JO, Ter-Pogossian MM (1974) The effects of changes in $\mathrm{PaCO}_{2}$ on cerebral blood volume, blood flow, and vascular mean transit time. Stroke 5:630-639

3. Powers WJ (1991) Cerebral hemodynamics in ischemic cerebrovascular disease. Ann Neurol 29:231-240

4. Powers WJ, Grubb RL, Raichle ME (1984) Physiological responses to focal cerebral ischemia in humans. Ann Neurol 16:546-552

5. Zaharchuk G, Mandeville JB, Bogdonov AA Jr, Weissleder R, Rosen BR, Marota JJ (1999) Cerebrovascular dynamics of autoregulation and hypoperfusion: an MRI study of CBF and changes in total and microvascular cerebral blood volume during hemorrhagic hypotension. Stroke 30:2197-2203

6. Sorensen AG, Copen WA, Ostergaard L, Buonanno FS, Gonzalez RG, Rordorf G, Rosen BR, Schwamm LH, Weiskoff RM, Koroshetz WJ (1999) Hyperacute stroke: simultaneous measurement of relative cerebral blood volume, relative cerebral blood flow, and mean tissue transit time. Radiology 210:519-527
7. Belliveau JW, Rosen BR, Kantor HL, Rzedzian RR, Kennedy DN, McKinstry RC, Vevea JM, Cohen MS, Pykett IL, Brady TJ (1990) Functional cerebral imaging by susceptibilitycontrast NMR. Magn Reson Med 14:538-546

8. Rosen BR, Belliveau JW, Buchbinder BR, McKinstry RC, Porkka LM, Kennedy DN, Neuder MS, Fisel CR, Aronen HJ, Kwong KK, Brady TJ (1991) Contrast agents and cerebral hemodynamics. Magn Reson Med 19:285-292

9. Rosen BR, Belliveau JW, Vevea JM, Brady TJ (1990) Perfusion imaging with NMR contrast agents. Magn Reson Med 14:249-265

10. Le Bihan D, Breton E, Lallemand D, Grenier P, Cabanis E, Laval-Jeantet M (1986) MR imaging of intravoxel incoherent motions: application to diffusion and perfusion in neurologic disorders. Radiology 161:401-407

11. Boxerman JL, Hamberg LM, Rosen BR, Weisskoff RM (1995) MR contrast due to intravascular magnetic susceptibility perturbations. Magn Reson Med 34:555-566

12. Villringer A, Rosen BR, Belliveau JW, Ackerman JL, Lauffer RB, Buxton RB, Chao YS, Wedeen VJ, Brady TJ (1988) Dynamic imaging with lanthanide chelates in normal brain: contrast due to magnetic susceptibility effects. Magn Reson Med 6:164-174

13. Stewart GN (1893) Research on the circulation time in organs and on the influences which affect it. J Physiol 15:1-89
14. Meier P, Zierler KL (1954) On theory of indicator-dilution method for measurement of blood flow and volume. J Appl Physiol 6:731-744

15. Weisskoff RM, Zuo CS, Boxerman JL, Rosen BR (1994) Microscopic susceptibility variation and transverse relaxation: theory and experiment. Magn Reson Med 31:601-610

16. Boxerman JL, Rosen BR, Weisskoff RM (1997) Signal-to-noise analysis of cerebral blood volume maps from dynamic NMR imaging studies. J Magn Reson Imaging 7:528-537

17. Ogawa S, Lee TM, Kay AR, Tank DW (1990) Brain magnetic resonance imaging with contrast dependent on blood oxygenation. Proc Natl Acad Sci USA 87:9868-9872

18. Ogawa S, Lee TM, Nayak AS, Glynn P (1990) Oxygenation-sensitive contrast in magnetic resonance image of rodent brain at high magnetic fields. Magn Reson Med 14:68-78

19. Lev MH, Kulke SF, Sorensen AG, Boxerman JL, Brady TJ, Rosen BR, Buchbinder BR, Weisskoff RM (1997) Contrast-to-noise ratio in functional MRI of relative cerebral blood volume with sprodiamide injection. J Magn Reson Imaging 7:523-527 
20. Perthen JE, Calamante F, Gadian DG, Connelly A (2002) Is quantification of bolus tracking MRI reliable without deconvolution? Magn Reson Med 47:61-67

21. Calamante F, Gadian DG, Connelly A (2002) Quantification of perfusion using bolus tracking magnetic resonance imaging in stroke: assumptions, imitations, and potential implications for clinical use. Stroke 33:1146-1151

22. Detre JA, Leigh JS, Williams DS, Koretsky AP (1992) Perfusion Imaging. Magn Reson Med 23:37-45

23. Detre JA, Alsop DC, Vives LR, Maccotta L, Teener JW, Raps EC (1998) Noninvasive MRI evaluation of cerebral blood flow in cerebrovascular disease. Neurology 50:633-641

24. Born AP, Rostrup E, Miranda MJ, Larsson HBW, Lou HC (2002) Visual cortex reactivity in sedated children examined with perfusion MRI (FAIR). Magn Reson Imaging 20:199-205

25. Ball WS Jr, Holland SK (2001) Perfusion imaging in the pediatric patient. Magn Reson Imaging Clin N Am 9:207-230

26. Tokumaru AM, Barkovich AJ, O uchi T, Matsuo T, Kusano S (1999) The evolution of cerebral blood flow in the developing brain: evaluation with iodine-123 iodoamphetamine SPECT and correlation with MR imaging. Am J Neuroradiol 20:845-852

27. Takahashi T, Shirane R, Sato S, Yoshimoto T (1999) Developmental changes of cerebral blood flow and oxygen metabolism in children. Am J Neuroradiol 20:917-922

28. Monakow C von (1900) Uber die projektions und die assoziationszentren im grosshirn. Monatsschr Psychiatrie 8:405-420

29. Craigie EH (1924) Changes in the vascularity in the brain stem and cerebellum of the albino rat between birth and maturity. J Comp Neurol 38:27-48

30. Kennedy C, Grave GD, Jehle JW, Sokoloff L (1970) Blood flow to white matter during maturation of the brain. Neurology 20:613-618
31. Kennedy C, Grave GD, Jehle JW, Sokoloff L (1972) Changes in blood flow in the component structures of the dog brain during postnatal maturation. J Neurochem 19:2423-2433

32. Gonzalez RG, Schaefer PW, Buonanno FS, Shwamm LH, Budzik RF, Rordorf G, Wang B, Sorensen AG, Koroshetz WJ (1999) Diffusion-weighted MR imaging: diagnostic accuracy in patients imaged within 6 hours of stroke symptom onset. Radiology 210:155-162

33. Kaufmann AM, Firlik AD, Fukui MB, Wechsler LR, Jungries CA, Yonas H (1999) Ischemic core and penumbra in human stroke. Stroke 30:93-99

34. Schlaug G, Benfield A, Baird AE, Siewert B, Lovblad KO, Parker RA, Edelman RR, Warach S (1999) The ischemic penumbra: operationally defined by diffusion and perfusion MRI. Neurology 53:1528-1537

35. Davis R, Bulkley G, Traystman R (1988) Role of oxygen free radicals in focal brain ischemia. In: Tomita M, Sawada T, Naritomi H, Weiss W-D (eds) Cerebral hyperemia and ischemia: from the standpoint of cerebral blood volume. Excerpta Medica, Amsterdam, pp 151-156

36. Keller E, Flacke S, Urbach H, Schild HH (1999) Diffusion- and perfusionweighted magnetic resonance imaging in deep cerebral venous thrombosis. Stroke 30:1144-1146

37. Tzika AA, Robertson RL, Barnes PD, Vajapeyam S, Burrows PE, Treves ST, Scott RM (1997) Childhood Moyamoya disease: hemodynamic MRI. Pediatr Radiol 27:727-735

38. Tsuchiya K, Inaoka S, Mitzutani Y, Hachiya J (1998) Echo-planar perfusion MR of Moyamoya disease. Am J Neuroradiol 19:211-216

39. Calamante F, Ganesan V, Kirkham FJ, Jan W, Chong WK, Gadian DG, Connelly A (2001) MR perfusion imaging in Moyamoya syndrome: potential implications for clinical evaluation of occlusive cerebrovascular disease. Stroke 32:2810-2816

40. Aronen HJ, Gazit IE, Louis DN, Buchbinder BR, Pardo FS, Weisskoff RM, Harsh GR, Cosgrove GR, Halpern EF, Hochberg FH, Rosen BR (1994) Cerebral blood volume maps of gliomas: comparison with tumor grade and histologic findings. Radiology 191:41-51

41. Knopp EA, Cha S, Johnson G, Mazumdar A, Golfinos JG, Zagzag D, Miller DC, Kelly PJ, Kricheff II (1999) Glial neoplasms: dynamic contrastenhanced T2*-weighted MR imaging. Radiology 211:791-798
42. Taylor JS, Tofts PS, Port R, Evelhoch JL, Knopp M, Reddick WE, Runge VM, Mayr N (1999) MR imaging of tumor microcirculation: promise for the new millenium. J Magn Reson Imaging 10:903-907

43. Aronen HJ, Glass J, Pardo FS, Belliveau JW, Gruber ML, Buchbinder BR, Gazit IE, Linggood RM, Fischman AJ, Rosen BR (1995) Echo-planar MR cerebral blood volume mapping of gliomas. Clinical utility. Acta Radiol 36:520-528

44. Rempp KA, Brix G, Wenz F, Becker CR, Gückel F, Lorenz WJ (1994) Quantification of regional cerebral blood flow and volume with dynamic susceptibility contrastenhanced MR imaging. Radiology 193:637-641

45. Sorensen AG, Rosen BR (1996) Functional MRI of the brain. In: Atlas-Scott W (ed) Magnetic resonance imaging of the brain and spine. Lippincott-Raven, Philadelphia, pp 1501-1545

46. Folkman J (1971) Tumor angiogenesis: therapeutic implications. N Engl J Med 285:1182-1186

47. Folkman J, Klagsbrun M (1987) Angiogenic factors. Science 235:442-447

48. Wesseling P, van der Laak JA, Link M, Teepen HL, Ruiter DJ (1998) Quantitative analysis of microvascular changes in diffuse astrocytic neoplasms with increasing grade of malignancy. Human Pathol 29:352-358

49. Zama A, Tamura M, Inoue HK (1991) Three-dimensional observations on microvascular growth in rat glioma using a vascular casting method. J Cancer Res Clin Oncol 117:396-402

50. Weisskoff RM, Zuo CS, Boxerman JL, Rosen BR (1994) Microscopic susceptibility variation and transverse relaxation: theory and experiment. Magn Reson Med 31:601-610

51. Dennie J, Mandeville JB, Boxerman JL, Packard SD, Rosen BR, Weisskoff RM (1998) NMR imaging of changes in vascular morphology due to tumor angiogenesis. Magn Reson Med 40:793-799 